REVESCO. Revista de Estudios Cooperativos ISSN: $1885-8031$

http://dx.doi.org/10.5209/REVE.62810

\title{
Efecto de las cooperativas exportadoras de café en el crecimiento de la economía solidaria en Colombia
}

Amanda Vargas Prieto ${ }^{1}$ y Diana Carolina Castañeda Guzmán ${ }^{2}$

Recibido: 18 de enero de 2018 / Aceptado: 19 de octubre de 2018

Resumen. El objetivo de este artículo es identificar el efecto generado por las cooperativas exportadoras de café en el crecimiento del sector solidario en Colombia. A través de una metodologia cualitativa se demuestra que las cooperativas exportadoras de café generan un efecto positivo en el crecimiento del sector solidario, y son representativas del mismo, puesto que son un motor de impulso para el sector agrícola, además de ser un actor fundamental en el comercio internacional, y en inversión de desarrollo social, mediante la inversión de educación formal, y otros programas de educación agrícola, por medio de los cuales se incentiva el buen uso del modelo cooperativo.

Palabras clave: Cooperativas; Cooperativas caficultoras; Economía solidaria; Colombia; Exportaciones.

Claves Econlit: J54; P13; Q13.

[en] Effects of the cooperative exporters of coffee in the growth of the solidarity economy in Colombia

\begin{abstract}
The objective of this article is to identify the effect generated by coffee exporting cooperatives in the growth of the solidarity sector in Colombia. Through a qualitative methodology it is shown that coffee exporting cooperatives have a positive effect on the growth of the solidarity sector, and are representative of it, since they are a driving force for the agricultural sector, besides being a fundamental player in International trade, and social development investment, through the investment of formal education, and other agricultural education programs, through which the good use of the cooperative model is encouraged.
\end{abstract}

Keywords: Cooperatives; Coffee cooperatives; Solidarity economy; Colombia; Exports.

Sumario. 1. Introducción. 2. Desarrollo. 3. Conclusiones. 4. Referencias bibliográficas.

Cómo citar: Vargas Prieto, A. y Castañeda Guzmán, D.C. (2019) Efecto de las cooperativas exportadoras de café en el crecimiento de la economía solidaria en Colombia. REVESCO. Revista de Estudios Cooperativos, Primer Cuatrimestre, No 130, pp. 213-234. DOI: 10.5209/REVE.62810.

$1 \quad$ Universidad de La Salle, Colombia

Dirección de correo electrónico: amvargas@unisalle.edu.co

2 Universidad de La Salle, Colombia

Dirección de correo electrónico: carito.castanedag@gmail.com 


\section{Introducción}

La producción agrícola y minera han sido históricamente la base del crecimiento económico de Colombia. Este es un país dedicado principalmente a la producción del sector primario, siendo sus principales productos de exportación el petróleo, carbón y café. Las exportaciones de dichos productos permitieron los primeros pasos en la inserción económica colombiana en el resto del mundo, abriendo camino a la internacionalización y el comercio internacional.

La internacionalización representa un reto para los países en vía de desarrollo, puesto que la incursión de las multinacionales afecta a las pequeñas y medianas empresas colombianas. En la búsqueda de alternativas para afrontar este desafío, se han encontrado soluciones como la constitución de cooperativas en las que grupos de personas forman organizaciones de propósito común y tienen como objetivo mejorar su calidad de vida (Melián y Campos, 2010; Monzón y Chaves, 2012). Las cooperativas, son un sistema de inclusión que contribuye a reforzar el desarrollo social y económico (Puentes y Velasco, 2009). El cooperativismo es una de las formas organizativas de la economía solidaria consideradas como una fuente de inclusión en la actividad económica en Latinoamérica, de acuerdo al debate sobre el desarrollo realizado por la Organización Internacional del Trabajo - OIT y la Alianza Cooperativa Internacional - ACI (2015). Sin embargo, la confianza en las cooperativas se ha visto deteriorada a través de los años y son percibidas como organizaciones con ánimo de lucro, orientadas a la evasión de impuestos y descargas de compromiso de carácter legal (Fajardo Martinez, 2010), lo cual ha generado desconfianza en la ciudadanía respecto a la información existente sobre las cooperativas y otras entidades del sector solidario. Las cooperativas representan el $4.9 \%$ del PIB nacional y cuentan con más de 5.9 millones de asociados, el cooperativismo se ha convertido en el nuevo motor económico del país, y se espera que su crecimiento sea mayor cada año (Portafolio, 2014), por lo que representa un tema interesante para investigar detalladamente y es pertinente contribuir en la construcción de un análisis específico del sector.

Dentro del marco Solidario Colombiano, se encuentra que las cooperativas representan el $68 \%$ de las organizaciones de economía solidaria, su sector más representativo es el financiero, seguido por el agrícola (CONFECOOP, 2015). Aunque en los últimos años el sector financiero es cada vez más significativo, las cooperativas agrícolas son un motor fundamental para el desarrollo económico del país. La investigación acerca de las cooperativas agrícolas es realizada solo por entidades oficiales tales como la Confederación de Cooperativas Colombianas (CONFECOOP) y la Superintendencia de Entidades Solidarias, por lo que ésta es limitada a informes periódicos sobre el panorama general del sector. Dentro de las cooperativas agrícolas se encuentra que los principales productos ofrecidos son: leche, café, algodón, aves, cerdo, banano, cereales, caña de azúcar, ganado vacuno, silvicultura, y flores, siendo el café uno de los gremios más significativos puesto que este representa el $4.8 \%$ de las exportaciones totales de 2014 (PROCOLOMBIA, 2015). En el informe presentado por el observatorio cooperativo en el 2008 se evidencia que son muy pocas las empresas líderes que impulsan modelos cooperativos de desarrollo eficiente basados en la cadena de consumo, adicionalmente se ha encontrado que las cooperativas ofrecen a las 
personas una forma alternativa de desarrollo, para aquellos dedicados a la producción agropecuaria (Observatorio Cooperativo, 2008).

$\mathrm{La}$ asociación de los productores colombianos ha creado importantes entidades como la Federación Nacional de Cafeteros de Colombia. Este sector es trascendental para la economía, por lo que es conveniente ejecutar un estudio preciso sobre el efecto generado en el crecimiento económico del sector gracias a las exportaciones de dicho gremio. Actualmente la investigación acerca de las cooperativas cafetaleras es reducida, y su información se encuentra limitada a la suministrada principalmente por la Federación Nacional de Cafeteros, limitándose a la recopilación de datos de producción de este producto. Esta problemática da origen a la pregunta de investigación que se pretende resolver en este artículo ¿Cuál es el efecto generado por las cooperativas exportadoras de café en el crecimiento del sector solidario?

Para seleccionar el periodo de estudio, se tuvo en cuenta que los datos encontrados en la Confederación de Cooperativas de Colombia se encuentran consolidados de forma organizada desde el año 2005, los datos de periodos anteriores son inconsistentes y poco confiables, y los datos encontrados de 2015 no son los definitivos y difieren entre las diferentes fuentes de información. Así, con el fin de obtener mejores y más precisos resultados se considera que el análisis de un periodo de 9 años comprendido entre los años 2005 al 2014 es idóneo para el desarrollo de esta investigación.

Para el desarrollo del primer objetivo: caracterización del sector solidario colombiano en los últimos 10 años, se utilizaron bases de datos del banco de la república sobre el IPC histórico, con el fin de deflactar los precios presentados en los informes anteriormente mencionados. Además, se usaron datos extraídos del Departamento Administrativo Nacional de Estadísticas (DANE), sobre el Producto Interno Bruto histórico y datos del empleo, con el fin de realizar un análisis comparativo sobre los ingresos de las organizaciones del sector cooperativo y los ingresos nacionales, y el empleo generado por el sector solidario dentro de las personas económicamente activas. Para el desarrollo del segundo objetivo, la identificación de las cooperativas exportadoras de café, primero se contextualizó el sector caficultor en Colombia a través de la evaluación el comportamiento del sector cafetalero en el comercio exterior, la evolución de las exportaciones entre el año 2004 y 2014, la participación del sector en la economía nacional, y las exportaciones de la Federación Nacional, luego se realizó una entrevista con el director de Desarrollo Cooperativo de la Federación Nacional de Cafeteros con el fin de conocer en mayor profundidad la acción de FEDECAFE, y de las cooperativas caficultoras. Por último se examinó la inversión en la educación de las cooperativas. Pues se considera que a través de la educacion la actividad cooperativa no sólo tiene como objetivo repercutir sobre los integrantes de la organización sino sobre el resto de la población (Carrasco, 2005; Martínez Charterina, 2015). Para la recolección de datos, se usaron fuentes como el DANE para la evaluación de la participación nacional del sector cafetero, el Banco Mundial para evaluar el comportamiento del sector en el marco internacional, la Federación Nacional de Cafeteros, para evaluar las exportaciones según exportador. Todo esto con el fin de realizar un análisis descriptivo sobre el comportamiento del sector en el periodo de estudio. Para el tercer objetivo, evaluar 
la participación de las cooperativas exportadoras de café en la generación de ingresos del sector solidario, se realizó un análisis estadístico comparativo, en el cual, se comparan los ingresos del sector solidario, con los ingresos por exportaciones de las cooperativas caficultoras. Para la recolección de datos de los ingresos del sector solidario se utilizaron los informes de CENICOOP en el periodo de estudio (2004-2014), y el informe de la supersolidaria de Estadísticas del sector solidario diciembre 2002 a diciembre 2015.

\section{Desarrollo}

\subsection{Antecedentes del cooperativismo en el crecimiento económico}

El Cooperativismo en la economía colombiana ha tomado gran importancia en los últimos años, convirtiéndose en un tema de interés para empresarios e investigadores, ya que es una alternativa de desarrollo (Fonseca et al. 2017), en los siguientes antecedentes de investigación se puede observar como el cooperativismo ha afectado de alguna forma a la economía y el desarrollo desde diferentes puntos de vista (Pastor, 2011).

Por ejemplo, en la investigación de Rodríguez Gaitán, Rueda Angarita, y Sánchez Brand (2010) se demuestra la gran contribución a la economía nacional, y la importancia de este sector para lograr una mejor distribución de las riquezas para las economías en desarrollo. Por otro lado, se concluye que la prevalencia del sector solidario se basa en el desarrollo de una regulación más adecuada, la adhesión a los principios de economía solidaria, y la determinación de ideas innovadoras para la creación de estrategias crecimiento de dicho sector. Esto quiere decir que por medio de una regulación correcta se pueden obtener mejores resultados en cuanto a la implementación de los principios y valores que rigen la economía solidaria, creando así mayores oportunidades para la promulgación de la misma, además se genera una distribución más adecuada de las riquezas que beneficia a la comunidad participante de dicho sistema económico.

Por otro lado dentro de las características básicas de la organización y gestión solidaria planteados por Dávila (2005), encontramos que las organizaciones solidarias están definidas por tres enfoques; asociativo, empresarial y educativa, que en conjunto propician el desarrollo de una cultura coherente con la propuesta solidaria, la democracia y la cooperación. Las organizaciones solidarias presentan una ventaja competitiva, la cual consiste en promover la satisfacción simultanea de necesidades económicas y sociales, asimismo genera el bienestar individual de los asociados (Dávila, 2005). Esto apoya la teoría de Porter (1991), sobre las ventajas competitivas, y afirma que la cooperación proporciona este tipo de ventaja para las organizaciones solidarias, dándole un valor agregado a las actividades que desarrollan, además del incremento de productividad, la ventaja competitiva en la economía de solidaridad, se presenta en el bienestar de los miembros.

Para López Castaño (2005) la solidaridad como forma organizada se ve reflejada en la constitución de cooperativas, como respuesta a los procesos de industrialización y una alternativa de desarrollo paralela al capitalismo. Un claro ejemplo del éxito de la cooperación como actividad económica, es el caso de Japón 
que a partir de la solidaridad organizacional creó modelos económicos que llevaron a fortalecer la economía nipona. En el caso colombiano el principal problema para las empresas solidarias es la gestión gerencial, la cual no se ha podido desligar del modelo capitalista y crear técnicas administrativas de planeación colectiva. La investigación realizada por López Castaño (2005), demuestra que el cooperativismo es un modelo eficiente no solo para economías emergentes, sino también para economías desarrolladas, y que el factor organizacional es eje clave para la ejecución correcta, y este es precisamente una de las dificultades en la economía de solidaridad colombiana.

La literatura demuestra que las cooperativas tienen gran importancia en el desarrollo rural, esta importancia no debe ser limitada a su influencia económica, también involucra aspectos sociales y culturales en la comunidad, y cuentan con algunas cualidades específicas que fomentan el desarrollo, la capacidad de acompañamiento en el desarrollo tecnológico, la relación con el territorio en donde llevan a cabo sus operaciones productivas, y la fomentación del empleo son algunas de las características que intervienen en el aumento de bienestar social (García, 1997; Sanchez Castañeda y Vargas Prieto, 2017; Matiz Piza et al. 2017).

En Costa Rica mediante el estudio dirigido por el Dr. Roberto Artavía, presidente del INCAE Business School, se identificó el impacto positivo de las cooperativas en su comunidad, en diferentes aspectos como el aumento de bienestar, la inversión en capital humano, el fomento de respeto por el ecosistema, el impulso de dinámicas de solidaridad, la certidumbre de los asociados y la convivencia comunitaria. Afirmando así que las comunidades con tradición cooperativa tienen mejores niveles de progreso social (INFOCOPP, 2015). Este tipo de investigación permite mostrar el aporte real del cooperativismo ante la comunidad, siendo el bienestar social, afectado positivamente mediante diferentes factores, como la inversión en el capital humano, esta inversión puede darse en campos como la educación, que se traduce en desarrollo, y crecimiento (Cáceres Gómez et al. 2017).

\subsection{Antecedentes del cooperativismo en el subsector exportador de café}

Para afrontar las problemáticas actuales de algunos países como pobreza, desempleo y falta de oportunidades, según la OIT y ACI (2015), las cooperativas son una alternativa para empezar nuevos negocios sin recurrir a un gran capital, por ejemplo PRODECOOP de Nicaragua, agrupa a unas 45 cooperativas integradas por más de 2420 familias las cuales producen y comercializan café de una forma sostenible generándoles así una calidad de vida estable (OIT y ACI, 2015). Generando así una alternativa para que dicha población pueda ser incluida en el sistema económico, y adicionalmente aumente su calidad de vida. En diferentes países de Latinoamérica el café es fuente principal de sus ingresos, y las cooperativas agrícolas representan una opción para que los pequeños productores puedan ser partícipes del desarrollo económico. Es por eso que Barriga Flores (2009), en Lima se interesa por evaluar el impacto de las cooperativas cafeteras en la vida de los asociados, en su investigación encuentra que el funcionamiento de las cooperativas puede llegar a ser más flexible de lo estipulado es sus reglamentos, esto provoca que el rango de acción de las cooperativas no se limite a sus 
asociados, afectando positivamente a la comunidad, generando mayor estabilidad económica, avances en investigación y mejoras en la infraestructura (Barriga Flores, 2009).

Además, las cooperativas agropecuarias representan en Latinoamérica un motor de desarrollo y prosperidad, en Brasil por ejemplo son las cooperativas agropecuarias las más significativas del sector, siendo las cooperativas cafetaleras la de mayor crecimiento, en el 2014, y las segundas exportadoras con $14.2 \%$ de las exportaciones totales de las cooperativas del mismo año, además Brasil produce más del 30\% de café a nivel mundial, siendo así las cooperativas cafetaleras uno de los pilares más fuertes de la economía Brasilera (Sistema OSB, 2015). A la red de la ICAO, pertenecen las cooperativas cafetaleras del Perú socias de la Junta Nacional del Café (JNC), las cuales en el 2015 exportaron 98 millones de dólares, lo que es igual al $23 \%$ del total de las exportaciones de café de Perú a diversos mercados de Estados Unidos, Asia y Europa (InfoRegión, 2015). Demostrando así la importancia del sector agrícola, específicamente de las cooperativas cafetaleras en el desarrollo económico de los diferentes países Latinoamericanos.

En Colombia, según la ACI, en el 2014 las cooperativas de caficultores les compraron a sus asociados 330 millones de kilos de café, un total de 2 billones de pesos inyectados en la economía, se destaca que las cooperativas caficultoras son aliadas estratégicas, que ejercen la garantía de compra de la cosecha de los productores, además de esto ofrecen posibilidades de créditos a los caficultores para la fertilización, renovación y siembra, también se han invertido 12.000 millones de pesos en programas educativos que benefician 1.400 estudiantes de diferentes universidades entre otros (El Colombiano, 2014). Esto es un indicador de que las cooperativas son una fuente de crecimiento y la creación de entidades como la ACI, impulsa la promulgación del cooperativismo en los países, dando la oportunidad a los asociados de aprovechar los beneficios de las buenas prácticas comerciales de estas formas organizativas, que representan una alternativa económica de fortalecimiento para los pequeños productores, que buscan un objetivo común y compromiso comunitario.

Por otro lado, en Colombia las cooperativas cafetaleras en el 2014 lograron renovar más de 9.000 hectáreas de las cuales el $93 \%$ de estas tierras están destinadas al cultivo de las variedades resistentes a la roya, además se benefició a más de 5.000 caficultores con financiamientos de 16.368 millones de pesos, y las exportaciones directas a doce países diferentes suman 184.531 sacos de café verde, equivalente al $59 \%$ de las ventas del año. La inversión social demandó recursos iguales a 1.730 millones de pesos, distribuidos en seguros de vida para los asociados y conyugues, descuentos de compra en fertilizantes y apoyo a la educación superior de los hijos de los asociados (El Colombiano, 2015). Este tipo de iniciativas ratifica la idea de que el cooperativismo no solo afecta a sus asociados, sino también al medio en que se desarrolla, con la renovación de tierras, todos los habitantes de la zona renovada tendrán beneficios ecológicos, además de beneficios generados por la inversión social, brindándole a la población oportunidades de educación y financiamiento en los cultivos. También, en el 2015, siete asociaciones de productores de café, cacao y frutas fueron elegidas por la Asociación Canadiense de Cooperativas (CCA) para un proyecto que beneficiará con 1.4 millones de dólares canadienses para fortalecer las organizaciones de 
productores agrícolas del Huila por medio de estrategias de acompañamiento financiero, social, administrativo y gerencial para el progreso económico de las cooperativas agrícolas (El Diario del Huila, 2015). Reflejando así el incentivo de inversión que pueden generar las diversas formas asociativas de la economía solidaria para el desarrollo social.

De esta forma se puede concluir que el sector solidario representa una ventaja competitiva, que satisface de forma simultánea las necesidades económicas y sociales, dentro de este sector en Colombia las cooperativas agrícolas fomentan el desarrollo rural y tecnológico del territorio en donde se lleva a cabo la actividad productiva, además la cooperativas cafetaleras colombianas han logrado grandes avances en la renovación de tierras, mediante la inversión social, y adquisición de inversión extranjera directa para el desarrollo. Se refleja entonces como la economía solidaria, y el cooperativismo, pueden traen beneficios tanto económicos y sociales (Vargas Prieto, 2018; 2017; Dávila et al. 2018; López et al. 2018), para los asociados y se considera que es importante evaluar el comportamiento de las cooperativas cafetaleras en Colombia.

\subsection{Caracterización del sector solidario colombiano en el periodo comprendido entre los años 2005 y 2014}

Existen tres diferentes sectores de la economía, el sector púbico, sector privado y el tercer sector, también conocido como el sector de economía solidaria, este tercer sector aparece por la necesidad de inclusión de las entidades que no son reconocidas como pertenecientes al sector privado o al sector público, y aun así son entidades privadas que no necesariamente son creadas con ánimo de lucro. Según la ley 454 de 1998 una organización de economía solidaria está caracterizada por ser personas jurídicas organizadas, con el fin de realizar actividades sin ánimo de lucro, en la cual los aportantes son a la vez los trabajadores y gestores, estas organizaciones tienen como objetivo satisfacer las necesidades de sus aportantes o miembros y desarrollar obras de servicio a la comunidad, mediante la producción, distribución, y manejo eficiente de los bienes y servicios.

El sector solidario colombiano está integrado por las organizaciones de economía solidaria, los organismos de apoyo y entidades estatales. Hay tres tipos diferentes de organizaciones de economía solidarias, las cooperativas, los fondos de empleados, y asociaciones mutuales. Son dos los organismos de apoyo existentes, el Consejo Nacional de Economía Solidaria - CONES, y el Fondo Nacional de Economía Solidaria - FONES. Las entidades estatales pertenecientes al sistema de la economía solidaria son tres, la Unidad Administrativa Especial de Organizaciones Solidarias- UAEOS, la Superintendencia de la Economía Solidaria, y el Fondo de Garantías para Entidades Cooperativas - FOGACOOP (SUPERSOLIDARIA, 2012). A continuación se presenta la caracterización de cada una de las organizaciones de economía solidaria. 


\subsubsection{Comportamiento de las organizaciones solidarias según el número de entidades}

Para analizar el comportamiento de las organizaciones del sector solidario se evalúa la composición del sector según el número de entidades y la variación anual del número de organizaciones. Así el análisis de gráficos junto con la revisión bibliográfica de los informes y artículos del comportamiento del tercer sector, nos genera una visión general del desempeño del sector en el periodo analizado. Los datos encontrados acerca de las diferentes organizaciones de economía solidaria son limitados, ya que a diferencia de los otros dos sectores de la economía, el tercer sector es un sector relativamente nuevo, y existen pocas entidades a cargo de la recopilación y análisis de dicho sector, entre ellas encontramos a la Supersolidaria, entidad a la cual son reportados los estados financieros de las entidades vigiladas, el DANE, departamento a cargo de las estadísticas nacionales y CENICOOP el centro de investigación cooperativa en Colombia. La distribución de las organizaciones según su categoría, cooperativa, asociaciones mutuales, o fondos de empleados, es un aspecto fundamental para la comprensión del funcionamiento del sector solidario, a continuación se presenta dicha distribución.

Gráfica. 1. Composición de las organizaciones solidarias por número de entidades (20052014)

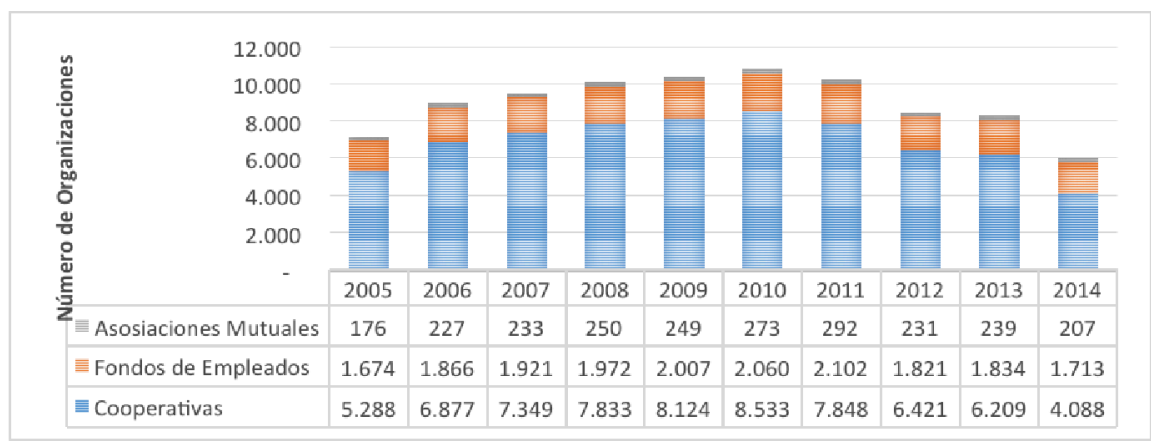

Fuente: elaboración propia, basado en datos de informes anuales de Cenicoop.

Esta gráfica muestra la composición de las organizaciones solidarias, según el número de entidades en el periodo comprendido entre los años 2005 y 2014, como se puede observar el mayor número de entidades en todos los años corresponde a las cooperativas, las cuales representan más del $60 \%$, del total de organizaciones solidarias, a estas la siguen los fondos de empleados, los que representan más del $20 \%$ en todos los años. Por último encontramos, a las asociaciones mutuales las que representan el 3\% del total de las organizaciones. De esta forma se ve como la mayoría de las entidades son cooperativas, esto muestra la preferencia de los colombianos por la conformación de estas organizaciones con el fin de satisfacer las necesidades mutuas y generar beneficios para sus asociados. El cooperativismo es el ente más representativo dentro del sector, por lo tanto su comportamiento es 
significativo para el análisis del comportamiento del sector solidario. El análisis de la variación porcentual en el número de organizaciones es un indicador general de comportamiento de las organizaciones de economía solidaria, a continuación en la gráfica 2 se puede ver dicho comportamiento según los datos presentados por la Supersolidaria en el consolidado de resúmenes financieros des año 2002 al 2015.

Gráfica. 2. Variación anual en el número de organizaciones de economía solidaria (20052014)

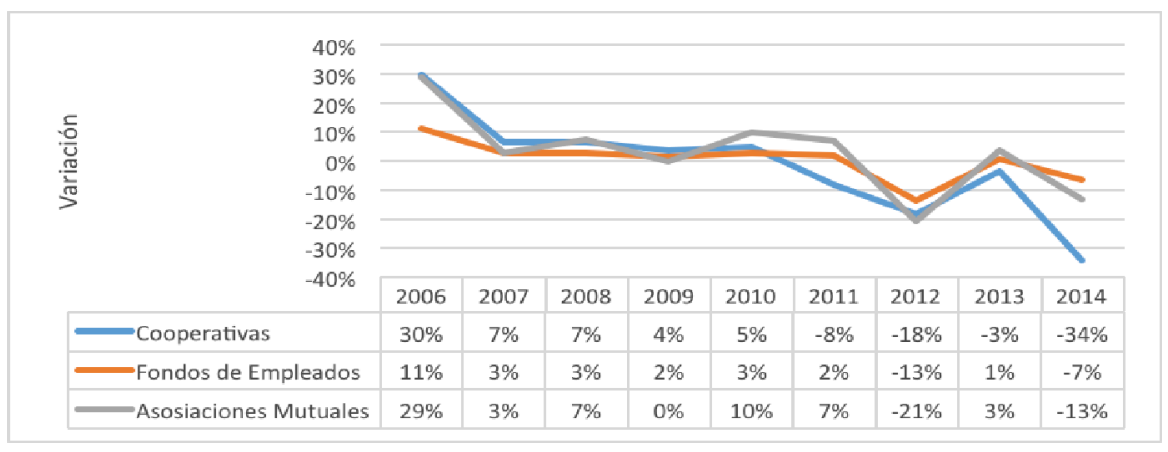

Fuente: elaboración propia, basado en datos de informes anuales de Cenicoop.

La gráfica 2 muestra la variación porcentual en el número de entidades de economía solidaria desde el 2005 hasta el 2014, se puede observar que los fondos de empleados son las organizaciones que menos ha variado en el número de organizaciones, las asociaciones mutuales presentan una variación negativa, siendo los periodos del 2004 al 2005 y 2013 a 2014 en donde han disminuido en mayor cantidad estas entidades, alcanzando hasta un $21 \%$. Las cooperativas son las organizaciones con la mayor variación, alcanzando su punto más bajo en el 2012, en donde alrededor de 1400 cooperativas dejaron de existir. Así se puede decir que en el periodo analizado a partir del 2011 existe una tendencia de variación negativa en el número de organizaciones, y las variaciones en el 2012 en las cooperativas son las más significativas, según el informe del Observatorio Cooperativo sobre el sector cooperativo del 2012, esta tendencia se debe a factores como las menores ventas del subsector lechero, la disminución en producción cafetera, la reducción de colocación de crédito y la reducción de las cooperativas de trabajo asociado (Observatorio Cooperativo, 2012). Algunos factores externos y las condiciones de mercado desfavorables de 2012 afectaron especialmente las cooperativas de actividad agropecuaria, las cuales no son ajenas a crisis de la zona euro, crisis que causó la recesión económica en dicho año, obligando así a los países europeos a disminuir sus importaciones provenientes de las economías latinoamericanas.

Adicionalmente, según el informe de Desempeño del sector Cooperativismo Colombiano de 2014 la implementación de medidas por los diferentes entes de control para regular el uso indebido del tipo de modelo económico solidario, en el que las entidades se encontraban haciendo intermediación laboral en lugar de la aplicación del modelo cooperativo, es la causa principal en la disminución del 
número de organizaciones de economía solidaria a partir del 2011 (Cenicoop y Confecoop, 2015). Mediante el análisis de gráficos y los informes presentados por las diferentes entidades de control del sector solidario, se puede concluir, que en general el sector solidario ha presentado en los últimos años un decrecimiento en cuanto al número de entidades, y aunque parezca un indicador negativo, es por el contrario la muestra de la importancia del sector, ya que el incremento del peso del tercer sector en la economía colombiana, llama la atención de las autoridades gubernamentales para generar un mayor control y vigilancia de las organizaciones solidarias que trae como consecuencia la acentuación de confiabilidad en el sector, factor fundamental para su crecimiento.

\subsubsection{Comportamiento de las organizaciones solidarias según empleados y asociados}

Las organizaciones de economía solidaria pueden ser evaluadas en su alcance a la población desde dos puntos de vista diferente. El primero por la cantidad de empleados de cada entidad, que representa a las personas que trabajan directamente con la organización, y desde la cantidad de asociados, quienes son aquellos que contribuyen económicamente, y adquieren derechos y deberes con la organización a la que pertenecen. Los empleados por su parte, para mayor entendimiento de la estructura solidaria, en el sector cooperativo, el cual es el más representativo, no sólo en su número de entidades como vimos anteriormente, sino también en el número de empleos generados, están divididos en dos grupos, el primero representa los empleados directos, es decir las personas que trabajan bajo el régimen laboral tradicional del sector cooperativo, y los empleados bajo el modelo de cooperativas de trabajo asociado. Los empleados del sector cooperativo representan más del 95\% del total de empleados de las organizaciones de economía solidaria en todos los años, los fondos de empleados representan entre el $1 \%$ y $2 \%$ del total de los empleados, y las asociaciones mutuales representan menos del $1 \%$, en cuanto al número de empleados.

Tabla. 1. Número de empleados según el tipo de organización (2005 - 2014)

\begin{tabular}{|llllllllllll} 
& $\mathbf{2 0 0 5}$ & $\mathbf{2 0 0 6}$ & $\mathbf{2 0 0 7}$ & $\mathbf{2 0 0 8}$ & $\mathbf{2 0 0 9}$ & $\mathbf{2 0 1 0}$ & $\mathbf{2 0 1 1}$ & $\mathbf{2 0 1 2}$ & $\mathbf{2 0 1 3}$ & $\mathbf{2 0 1 4}$ \\
\hline $\begin{array}{l}\text { Coop. } \\
\text { (R.T) }\end{array}$ & 94.021 & 114.924 & 111.951 & 123.643 & 138.047 & 139.703 & 138.549 & 126.696 & 127.014 & 127.296 \\
$\begin{array}{l}\text { Coop. } \\
\text { (T.A) }\end{array}$ & & & & & & & & & & & \\
\hline Fondos & 6.132 & 7.560 & 451.869 & 500.450 & 537.859 & 559.118 & 610.526 & 482.168 & 386.138 & 327.533 & 120.022 \\
Mutuales & 2.044 & 2.520 & 3.720 & 4.071 & 4.406 & 4.758 & 4.890 & 4.130 & 4.388 & 4.356 \\
\hline Total & 455.462 & 576.873 & 624.801 & 673.715 & 705.977 & 760.436 & 631.139 & 522.614 & 464.707 & 256.789 \\
\hline
\end{tabular}

Fuente: elaboración propia, basado en datos de informes anuales de Cenicoop.

La disminución en el número de empleados a través del tiempo se debe, al igual que la variación negativa de número de organizaciones, a factores como el 
incremento de vigilancia, en las entidades que hacen mal uso del modelo cooperativo, por lo que muchas de las organizaciones han sido controladas.

Gráfica. 3. Empleo generado por el sector solidario, empleo generado por otros sectores y tasa de desempleo (2005-2014)

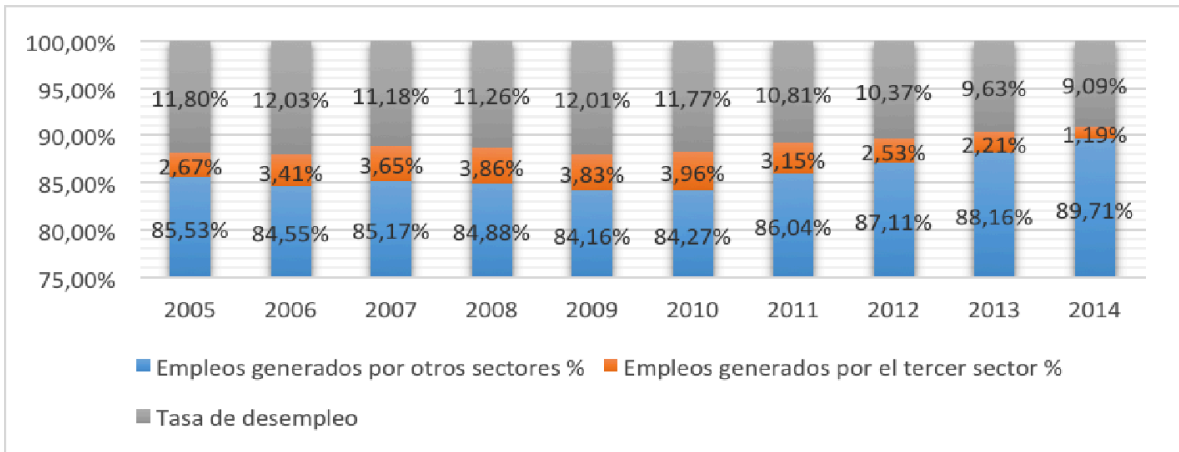

Fuente: elaboración propia, basado en datos de informes anuales de Cenicoop.

Mediante el análisis de la gráfica 3, se puede observar el porcentaje de empleados del sector solidario, el porcentaje de empleos generado por otros sectores, y la tasa de desempleo nacional, teniendo en cuenta que la población total de la gráfica la población económicamente activa, es decir la población que se encuentra en edad de trabajar y que se encuentra empleada o buscando trabajo, y la tasa de desempleo es igual a la cantidad de personas económicamente activos que se encuentran sin empleo sobre la población económicamente activa. Esta gráfica muestra como la tasa de desempleo nacional ha disminuido a través de los años del periodo de estudio, y a su vez como a partir del 2011 el porcentaje de empleos totales generados por el tercer sector se ha visto afectado negativamente, llegando al 2014, en el que del total de los empleados colombianos el $1.19 \%$, son pertenecientes al sector solidario. Así podemos ver que el sector solidario en general ha perdido porcentaje de participación en el empleo colombiano. Por otro lado los asociados a las organizaciones de economía solidaria muestran una tendencia diferente, un asociado es aquella persona que se adhiere voluntariamente, participa en las actividades de la cooperativa, y puede efectuar actos de decisión y elección, además debe cumplir con las decisiones de los órganos de administración. 
Gráfica. 4. Número de asociados según tipo de organización (2005-2014)

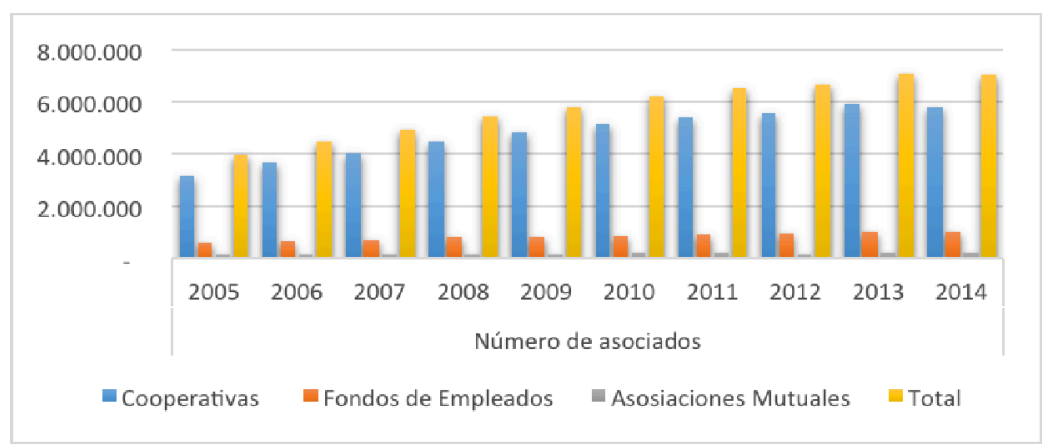

Fuente: elaboración propia, basado en datos de informes anuales de Cenicoop.

El número de asociados es considerado un indicador importante ya que, según el informe del desempeño cooperativo del 2012, se parte de la hipótesis de que cada asociado puede tener un núcleo familiar de 3 personas, así que los beneficio alcanzados por las organizaciones solidarias podrían incrementar 3 veces (Cenicoop, 2013). De esta forma se puede decir que en el 2005 cuando el número de asociados era de 3.980.000, y el número de habitantes en el país era de 41.686.000, el $9.55 \%$ de la población era parte de alguna organización solidaria como asociado, y en el 2014, con un número de asociados de 7.026.021 y una población total de 46.296 .000 , el $15.18 \%$ de la población colombiana obtiene beneficios del tercer sector. Bajo la hipótesis presentada con Cenicoop 2013, se podría decir que el alcance que pueden tener las organizaciones solidarias ascienden al $28.64 \%$ del total de la población colombiana en el 2004, y para el 2014 , este porcentaje es del $45.53 \%$ del total de la población.

Los beneficios que se obtienen por ser parte de entidades solidarias, en especial de las cooperativas, se generan gracias a la capacidad que tienen las entidades de trabajar paralelamente en dos actividades, la primera, de satisfacción de necesidades de los asociados, y la segunda, el desarrollo continúo para enfrentar los retos de la economía de mercado (Cenicoop, 2013). Esto genera un sistema sostenible, competitivo y rentable, que se traduce en la mejora de calidad de vida de sus asociados. Según el análisis de estos dos indicadores, empleo y número de asociados, se puede concluir que la disminución en la cantidad de empleados no se debe a la pérdida de interés por parte de los colombianos en el tercer sector, sino por los esfuerzos de generar un mayor control para que se cumplan los objetivos reales del modelo de economía solidaria, eso se corrobora mediante en incremento de número de asociados creciente en el periodo analizado, lo que indica aumento de interés de los colombianos por acceder a los diferentes beneficios que ofrecen las organizaciones solidarias 


\subsection{Identificación de las cooperativas exportadoras de café colombianas, entre los años 2005 y 2014}

Para determinar la verdadera importancia de las cooperativas cafetaleras en la economía solidaria, primero se dará una breve contextualización del sector cafetalero colombiano en el comercio exterior y su participación en la economía nacional.

\subsubsection{Las exportaciones de café colombianas en el mundo}

Para Colombia el café es un producto que nos identifica, y sus aportes al desarrollo económico son de gran importancia, este producto ha logrado estabilizar a través del tiempo el crecimiento económico pese a las recurrentes crisis de precios en el mercado internacional, también ha ayudado al fortalecimiento del mercado interno por medio de la generación de empleo, además de ayudar a la integración de las regiones con la apertura de vías y estimular la economía agrícola, sosteniendo el modelo primario-exportador por más de un siglo (Machado, 2001). A continuación se observa la participación de las exportaciones de café colombianas a nivel mundial desde el año 2004 al 2014.

Gráfica. 5. Participación en las exportaciones de café por país de origen.

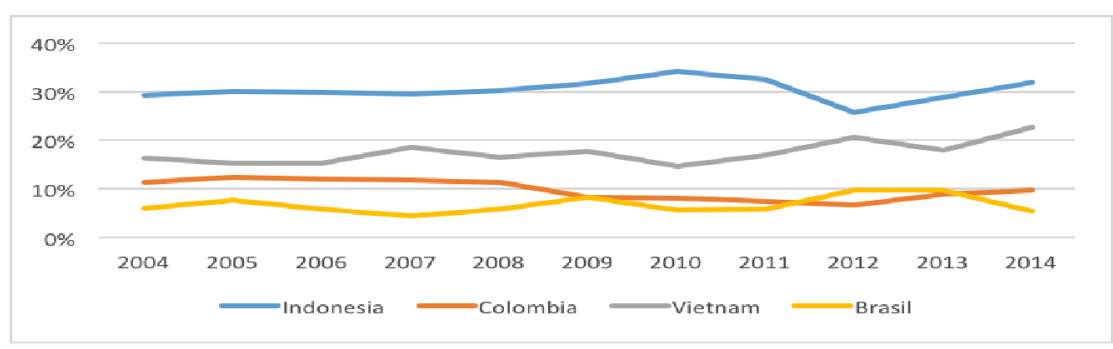

Fuente: elaboración propia, basado en datos de (C) International Coffee Organization.

Como se observa en la gráfica, la cual recopila la información de la participación en las exportaciones por país de origen por sacos de $60 \mathrm{~kg}$ de café verde, de los cuatro exportadores más grandes a nivel mundial, Colombia es uno de los países más importantes en cuanto a las exportaciones de café a nivel mundial, ubicándose como el tercer país exportador en el periodo de 2004 al 2009, disminuyendo su participación en las exportaciones hasta el año 2012, en donde se da una recuperación progresiva (gráfica 5). Esto demuestra la importancia de la producción de café colombiano a nivel internacional, manteniéndonos dentro de los 4 exportadores más grandes durante diez años, y se observa una tendencia al alza en los últimos años, aunque el 2016 se enfrenta con la amenaza del fenómeno del Niño y de la Niña, los cuales pueden afectar de forma negativa a la producción (El País, 2016). 


\subsubsection{Las exportaciones de café a nivel nacional}

De acuerdo a los datos históricos de exportaciones presentados por el DANE, los bienes tradicionales son indispensables en el desarrollo de comercio internacional, los bienes tradicionales son, el café, petróleo y sus derivados, carbón y ferroníquel, estos productos representan más del $50 \%$ de las exportaciones totales nacionales, por lo que son productos fundamentales para la economía colombiana.

Gráfica. 6. Participación porcentual de las exportaciones de café, en las exportaciones totales y tradicionales.

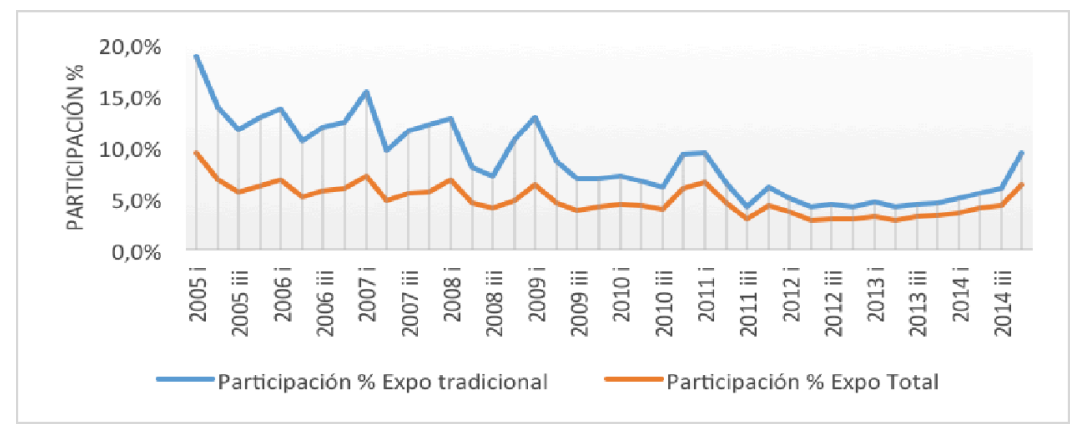

Fuente: elaboración propia, basado en datos del DANE

En el periodo de estudio analizado, el café representa en el 2005 el 18.9\% del total de las exportaciones tradicionales, alcanzando su punto más bajo en el 2011, con una participación del $4.1 \%$, y recuperándose lentamente en los últimos años hasta llegar a un $9.4 \%$ de la participación. En cuanto a la participación de las exportaciones de café sobre el total de exportaciones, encontramos que para el 2005 era del $9.4 \%$, su punto más bajo fue del $3 \%$, y en el 2014 , se recupera alcanzando un $6.3 \%$ (gráfica 6). Esto corrobora la importancia de la producción de café para la economía colombiana, llegando a generar ingresos por exportaciones en el 2014 por \$2.473.248 miles de dólares FOB. La caída en las exportaciones del 2011 se explica por el fuerte periodo que sufrió en general el sector agrícola, por las adversidades climáticas que arrasaron con cientos de cultivos, y desestabilizaron las productoras de café.

Con esto se puede corroborar que aun Colombia es un país dedicado a la producción del sector primario y aunque su participación en las exportaciones en el sector tradicional han disminuido, su participación en las exportaciones nacionales no muestra una gran variación, y se demuestra la importancia para la economía nacional, ya que los ingresos por exportaciones de café representan aproximadamente el $5 \%$ de los ingresos totales a través de los años estudiados. 


\subsubsection{Participacion de las cooperativas en las exportaciones de café en Colombia}

En cuanto a las exportaciones de café nacional, según la Federación Nacional de Cafeteros (2015), quien presenta esta información en miles de millones de dólares constantes a precios del 2015 , se observa que las exportaciones realizadas por el Federación Nacional de Cafeteros oscilan entre el 20\% y 30\% de las exportaciones totales en el periodo de estudio y las demás exportaciones de café son realizadas por organizaciones particulares (gráfica 7).

Gráfica. 7. Exportaciones de café realizadas por la Federación Nacional de Cafeteros y otros particulares.

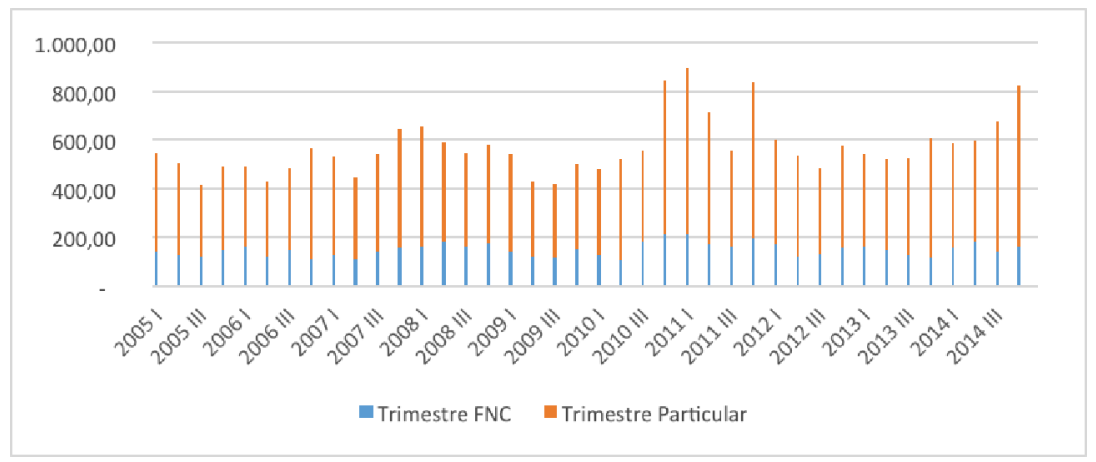

Fuente: elaboración propia, basado en datos de la Federación Nacional del Café.

La federación nacional de cafeteros representa en el 2015 a 33 cooperativas de caficultores colombianos, que representan 82.000 caficultores, esto quiere decir que los datos presentados por la Federación son considerados representativos para la explicación de la dinámica del subsector cooperativo de café.

\subsection{Las cooperativas según inversión en educación}

Según la ley 863 de 2003, para que las cooperativas sean exentas del pago de impuesto de renta, estas deben invertir el $20 \%$ de sus excedentes, en diferentes programas de educación formal, durante el año siguiente. Esta inversión es gestionada por el Ministerio de Educación, y destinada a diferentes áreas como educación superior, educación media, y básica. A continuación vemos la inversión en educación realizada por las cooperativas en el periodo de estudio. 
Gráfica. 8. Inversión en educación formal 2005 - 2013

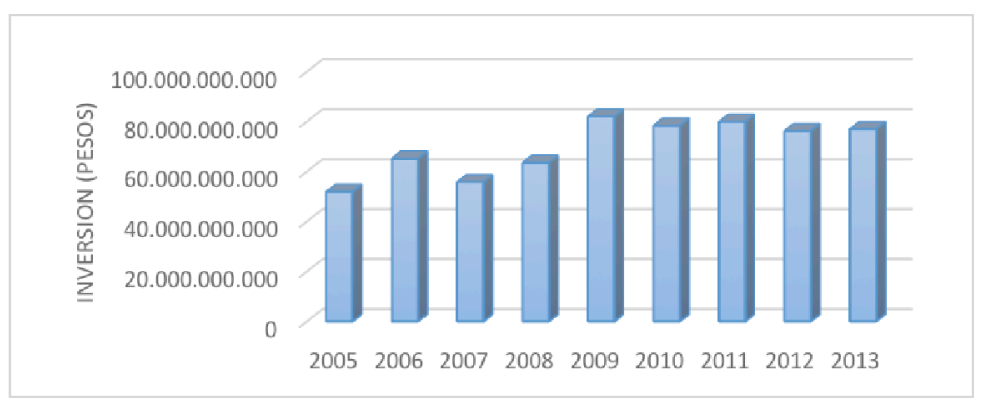

Fuente: elaboración propia, basado en datos de Informes anuales de Cenicoop.

La gráfica 8 muestra la inversión total que el cooperativismo ha hecho en programas de educación formal, se evalúa este rubro por que la educación es un claro indicador de desarrollo, y muestra como esta cifra es ascendente, llegando a alcanzar más de 75.000.000.000 de pesos en el 2013, con la inversión realizada por las cooperativas en educación se han beneficiado más de 1 millón de jóvenes colombianos hijos de asociados.

\subsection{Evaluacion de la participación de los ingresos por exportaciones de las cooperativas cafetaleras en los ingresos del sector solidario.}

Las exportaciones de café realizadas por las cooperativas de caficultores, son un indicador de crecimiento económico para dicho subsector, por eso es indispensable evaluar su comportamiento y participación en los ingresos del sector solidario para el desarrollo de esta investigación.

Gráfica. 9. Participación de los ingresos por exportaciones de las cooperativas en el sector solidario (2005-2014)

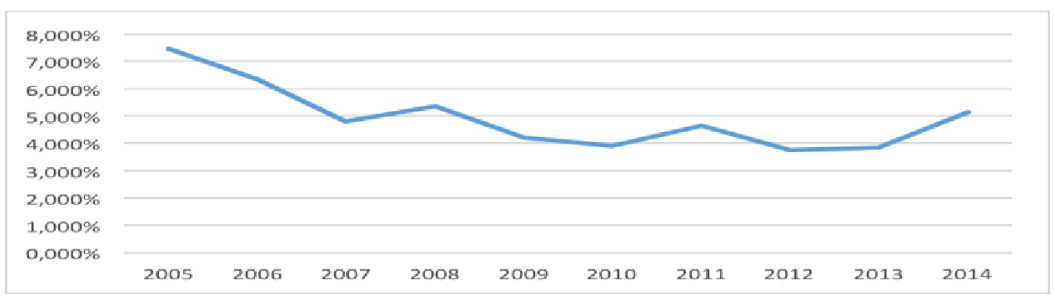

Fuente: elaboración propia, basado en datos de la Supersolidaria, el Banco de la república, y el Fondo nacional del Café.

Como se observa en la gráfica 9, la participación de los ingresos por exportaciones de café para las cooperativas, representan aproximadamente el 5\% de los ingresos del sector solidario a lo largo del periodo de estudio, esto quiere decir que los ingresos por exportaciones de los caficultores son responsables de 
generar más de 886 mil millones de pesos, lo que es equivalente en el 2014 al 0.2 de los ingresos totales del país. Esto es un indicador económico acerca del tamaño de este subsector, teniendo en cuenta que este análisis se hace tomando como muestra a las 33 cooperativas de caficultores pertenecientes al Fondo Nacional del Café, se considera que las cooperativas exportadoras de café son de alta relevancia para el sector solidario, ya que tan solo 33 entidades generan en el 2014 más del $5 \%$ de los ingresos del tercer sector.

Actualmente las exportaciones de café incrementaron en un $6 \%$ con respecto al primer trimestre de 2015 , pero estas disminuyeron en un $8 \%$ con respecto a abril del 2016, este comportamiento se le atribuye al fenómeno del Niño, el cual ha afectado de manera negativa al café excelso producido en el país (Redacción Economía y Negocios, 2016). Esto indica que a pesar de la disminución de las exportaciones, debido a la producción de café de menor calidad, la producción total de café se encuentra en aumento.

Gráfica. 10. Variación porcentual en los ingresos del tercer sector y las cooperativas de caficultores (2005-2014).

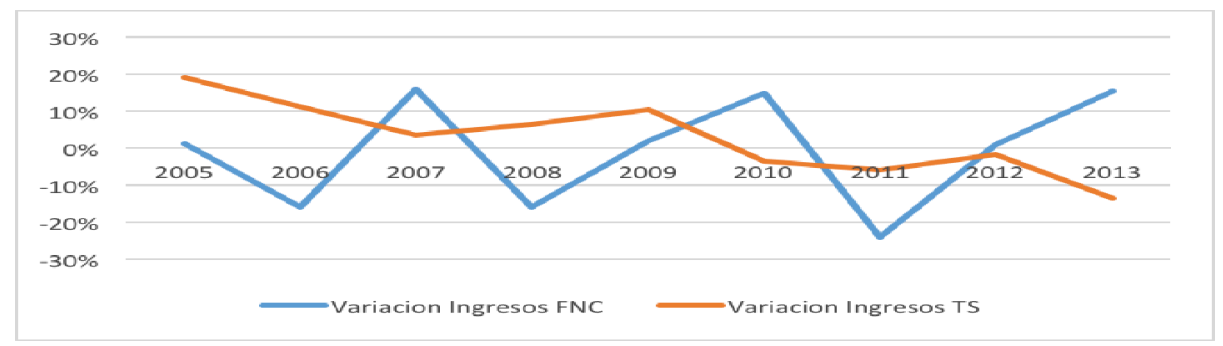

Fuente: elaboración propia, basado en datos de la Supersolidaria y el Fondo nacional del Café.

En la gráfica 10 se observa la variación porcentual de los ingresos del tercer sector y de las cooperativas exportadoras de café en el periodo de estudio, las cooperativas exportadoras de café tienen un comportamiento más dinámico, manteniendo su variación entre el $15 \%$ y $-20 \%$, por otro lado el sector solidario presenta una variación negativa constante en los últimos 3 años, reduciendo cada vez más sus ingresos totales. Esto nos indica que los ingresos del tercer sector no son directamente proporcionales a los ingresos por exportaciones de los caficultores, y se debe en gran parte a que el sector solidario está compuesto principalmente por entidades financieras, y su comportamiento es independiente al comportamiento del sector agrícola exportador. El Fondo Nacional de Café, junto al gobierno nacional adoptaron nuevas medidas para facilitar la exportación de pequeñas cantidades de café, esto con el fin de impulsar las exportaciones con destino a pequeños importadores, dicha medida causó un incremento del 74\% en el registro de exportadores en mayo del 2016, esta medida permite la exportación de pequeñas cantidades por medio de empresas de transporte internacional y mensajería expresa, y el alcance a nuevos clientes de forma directa ( Federación 
Nacional de Cafeteros, 2016). Esto permite a las familias de caficultores tener mayores ingresos por medio de la exportación de café directa.

Así se puede concluir que los ingresos por exportaciones de las cooperativas de caficultores son indispensables para el sector solidario ya que representan más del $5 \%$ de los ingresos del tercer sector en el periodo de estudio, y aunque estas no tengan una relación directamente proporcional, si son fuente de estabilidad económica, y sus esfuerzos por fortalecer el sector son ejemplo a seguir no solo por las entidades solidarias, sino también por las entidades privadas.

\section{Conclusiones}

En Colombia la economía de solidaridad ofrece alternativas para la inclusión en la actividad económica a la población más vulnerable, por medio del uso de diferentes herramientas como los fondos de empleados, las asociaciones mutuales y el cooperativismo, las cuales brindan la oportunidad de acceder a beneficios de la economía solidaria, como seguridad social, acceso a programas de desarrollo, educación, créditos, entre otros.

El sector solidario es un sector fundamental para la generación de empleo nacional, este ha sido responsable de generar el $1.19 \%$ de empleos para colombianos en el 2014, aunque antes del 2011 este porcentaje era mayor, y se ha visto afectado por los intentos gubernamentales de mejorar la implementación del modelo solidario. Los asociados son el motor de funcionamiento de las entidades de la economía solidaria, en el periodo de estudio este ha presentado un crecimiento constante, el número de asociados es considerado un indicador importante ya que, según el informe del desempeño cooperativo del 2012, se parte de la hipótesis de que cada asociado puede tener un núcleo familiar de 3 personas, así que los beneficio alcanzados por las organizaciones solidarias podrían incrementar 3 veces. En el 2005 cuando el número de asociados era de 3.980.000, y el número de habitantes en el país era de 41.686.000, el 9.55\% de la población era parte de alguna organización solidaria como asociado, y en el 2014, con un número de asociados de 7.026.021 y una población total de 46.296.000, el $15.18 \%$ de la población colombiana obtiene beneficios del tercer sector. Bajo la hipótesis presentada por Cenicoop 2013, se podría decir que el alcance que pueden tener las organizaciones solidarias asciende al $28.64 \%$ del total de la población colombiana en el 2004, y para el 2014, este porcentaje es del $45.53 \%$ del total de la población. Se puede concluir que la disminución en la cantidad de empleados no se debe a la pérdida de interés por parte de los colombianos en el tercer sector, sino por los esfuerzos de generar un mayor control para que se cumplan los objetivos reales del modelo de economía solidaria, eso se corrobora mediante en incremento de número de asociados creciente en el periodo analizado, lo que indica un aumento de interés de los colombianos por acceder a los diferentes beneficios que ofrecen las organizaciones solidarias. El cooperativismo es el modelo más representativo en la economía solidaria colombiana, las cooperativas representan más del $60 \%$ del total de organizaciones solidarias, a estas la siguen los fondos de empleados, los que representan más del $20 \%$ en todos los años, por último encontramos, a las asociaciones mutuales las que representan el 3\% del total de las organizaciones. 
Dentro de las cooperativas son las financieras las más representativas, seguidas por las dedicadas a la producción y comercialización agrícola. Las cooperativas de caficultores, las cuales pertenecen a las agrícolas, son un eje fundamental no solo para la economía solidaria, sino también para la economía nacional, el café es uno de los productos más importantes para el comercio internacional, Colombia es el cuarto exportador a nivel mundial encargado de realizar el $10 \%$ de las exportaciones, y a nivel nacional el café representa en promedio el $5 \%$ de las exportaciones totales, hecho que confirma la importancia de la producción y comercialización de este producto para la economía nacional. Las cooperativas de caficultores representan el en promedio el $30 \%$ de las exportaciones de café realizadas en el país, de esta forma se concluye que el 3\% de las exportaciones de café realizadas en el mundo son hechas por caficultores colombianos. Las cooperativas no sólo son responsables por las exportaciones, también influyen en el desarrollo en diferentes ámbitos, uno de ellos es la inversión en la educación, el $20 \%$ de los excedentes del cooperativismo deben ser destinados a proyectos de educación formal, en el 2005 el número de estudiantes beneficiados era de 486.417 y en el 2011 este número asciende a 1.585.642. Las cooperativas de caficultores en el 2014 invirtieron 7.900.000.000 pesos en el ICETEX, los cuales han beneficiado a 1700 jóvenes hijos de asociados, permitiéndoles acceder la educación superior formal. Así se concluye que la función de las cooperativas en el desarrollo social es indispensable, ya que estos beneficiarios son parte de la población perteneciente al sector agrícola, y es fundamental invertir en su educación para generar en el futuro mejor y mayores beneficios para dicho sector. El Factor C, es una característica fundamental del cooperativismo, dicho factor es la explicación de cómo una sociedad que se asocia para la satisfacción de sus necesidades, puede llegar a tener incluso mejores resultados a los obtenidos con el modelo clásico neo liberal. Las cooperativas, en donde la solidaridad se convierte en una fuerza productiva, traen como resultado el aumento en la eficiencia, el producto, y la productividad de las actividades económicas. Además, es un factor que suple las limitaciones de los otros factores de producción, generando un valor agregado a la economía. Es así como las 33 cooperativas pertenecientes a la federación nacional del café producen más del 50\% de café con un factor diferenciador, incrementando los ingresos de las familias caficultoras, y facilitando una mejora en la calidad de vida, además del acceso a créditos sin intereses por el primer mes para la mejora de sus cultivos, y programas de educación acerca del buen gobierno y gestión cooperativa. Todo esto en conjunto genera un valor agregado para los caficultores, que mediante la cooperación han logrado incrementar la producción en los últimos años, además de incrementar sus excedentes, los cuales son fuente primordial de inversión en la educación. A pesar de la disminución de ingresos del sector solidario en el periodo estudiado por la intervención del gobierno en entidades que hacían mal uso del modelo cooperativo, el sector exportador cooperativo ha mantenido un crecimiento constante, lo que representa que el sector de caficultores es fiel a los objetivos del cooperativismo y es digno ejemplo de desarrollo nacional. Esto permite dar respuesta a la pregunta de investigación ¿Cuál es el efecto generado por las cooperativas exportadoras de café en el crecimiento del sector solidario?, Las cooperativas exportadoras de café, generan un efecto positivo en el crecimiento sector solidario, y son representativas del mismo, puesto que son un motor de 
impulso para el sector agrícola, además de ser ente fundamental en el comercio internacional, y en inversión de desarrollo social, mediante la inversión de educación formal, y otros programas de educación agrícola, por medio de los cuales se incentiva el buen uso del modelo cooperativo.

\section{Referencias bibiliográficas}

BANCO DE LA REPÚBLICA. (Mayo de 2016) www.banrepcultural.org.

Barriga Flores, P. R. (2009) Funcionamiento de Cooperativas Cafetaleras y su impacto en la vida familiar de sus asociados: casos de familias de dos sectores de la provincia de la Convención vinculadas a la Cooperativa Maranura y a la central de cooperativas COCLA. Lima: Pontificia Universidad Católica del Perú.

Cáceres Gómez, L.S., Vargas Prieto, A. y Pedrozo Ruidíaz, L.M. (2017) Producción científica de la universidad de la Salle sobre economía solidaria 1974-2015. Revista Universitaria Ruta, [S.1.], $\mathrm{N}^{\mathrm{o}}$ 1, Vol. 19. ISSN 0719-5990. Disponible en: http://revistas.userena.cl/index.php/ruta/article/view/866. DOI: http://dx.doi.org/10.15433/ruta.v18i1.866.

Carrasco, I. (2005) La ética como eficiencia: la responsabilidad social en las cooperativas de crédito españolas. CIRIEC-España, Revista de Economía Pública, Social y Cooperativa, $\mathrm{N}^{\mathrm{o}}$ 53, pp. 351-367.

CENICOOP Y CONFECOOP (2015) Desempeño del sector cooperativo colombiano 2014. Bogotá:

https://confecoop.coop/images/informes_anuales/ReporteSectorCooperativo2014.pdf.

CENICOOP (2013) Informe de desempeño cooperativo 2012. Bogotá: Cenicoop. Confecoop.

CONFECOOP (2015) Desempeño del Sector Cooperativo 2014. Confederación de Cooperativas.

Dávila, R. (2005) Las características básicas de la organizacion y gestión solidaria. Bogotá: Universidad Javeriana de Colombia.

Dávila, R., Vargas, A., Blanco, L., Roa, E., Cáceres, L.E. y Vargas, L.A. (2018) Características de la economía solidaria colombiana. Aproximaciones a las corrientes influyentes en Colombia, CIRIEC-España, Revista de Economía Pública, Social y Cooperativa, № 93, pp. 85-113, DOI: 10.7203/CIRIEC-E.93.10327.

DIARIO DEL HUILA (02 de Abril de 2016). www.diariodelhuila.com. Obtenido de http://www.diariodelhuila.com/economia/huila-recibira-millonaria-inversion-paracreacion-y-fortalecimiento-de-empresas-solidarias-cdgint 20160402085044153.

EL COLOMBIANO (13 de 04 de 2015) www.aciamericas.com. Obtenido de http://www.aciamericas.coop/Colombia-cooperativas-compraron-35.

EL COLOMBIANO (8 de 12 de 2014) www.aciamericas.com.

EL DIARIO DEL HUILA (3 de 06 de 2015) www.aciamericas.coop. Obtenido de http://www.aciamericas.coop/La-Asociacion-Canadiense-de-Cooperativas-invierte-encooperativas-agropecuarias.

EL PAÍS (7 de Febrero de 2016) www.elpais.com.co. Obtenido de http://www.elpais.com.co/elpais/economia/noticias/volvio-buena-hora-cafe-colombiano.

Fajardo Martinez, C. E. (2010) RSE: Una reflexión desde la gestión, lo social y lo ambiental. Bogotá: Universidad Nacional. 
Fonseca, V., Contreras, L., Porras, L. y Vargas Prieto, A. (2017) Estado del arte sobre el desarrollo rural en Colombia. Revista CIFE, ISSN: 0124-3551, No 30, pp. 121-148. http://revistas.usta.edu.co/index.php/cife/article/view/3892.

García, A. M. (1997) Cooperativismo y Desarrollo Rural. REVESCO. Revista de Estudios Cooperativos, $\mathrm{N}^{\mathrm{0}}$ 63, pp. 63-77.

Gonzáles Meyer, R. y Richards, H. (2012) Hacia otras economías, Critica al paradigma dominante. LOM Ediciones.

INFOCOPP (27 de 10 de 2015) www.aciamericas.com. Obtenido de http://www.aciamericas.coop/Costa-Rica-las-comunidades-con-tradicion-cooperativatienen-mejores-niveles-de.

INFOREGIÓN (10 de 12 de 2015) www.aciamericas.com. Obtenido de http://www.aciamericas.coop/Cooperativas-cafetaleras-de-Peru-exportaron-por-98millones-de-dolares.

López Castaño, S. (2005) La solidaridad como fundamento de organizaciones empresariales emergentes. Una mirada a las cooperativas de Manizales. Manizales: Universidad Nacional de Colombia.

López, E. P., Martínez, L. M., Martínez-Cañas, C. A. y Vargas-Prieto, A. (2018) Desarrollo rural y envejecimiento: caso de estudio municipio de Chinavita, Boyacá, Colombia. Rev.investig.desarro.innov, $\quad \mathrm{N}^{\mathrm{o}} \quad 8, \quad$ Vol. 2, pp. 193-206. DOI: 10.19053/20278306.v8.n2.2018.7959.

Machado, A. (2001) El café en Colombia a princios del siglo XX. En Desarrollo economuco y social en Colombia : siglo XX (pág. 77). Bogotá: Universidad Nacional de Colombia.

Martínez Charterina, A. (2015) Las cooperativas y su acción sobre la sociedad. REVESCO. Revista de Estudios Cooperativos, Primer Cuatrimestre, No 117, pp. 34-49. DOI: 10.5209/rev_REVE.2015.v117.48144.

Matiz Piza, M., Guillen Cruz, M.S., Cano Parra, D.A. y Vargas Prieto, A. (2017) La economía solidaria en la mejora de la producción y comercialización de los dulces típicos de San Basilio de palenque. En: Colombia, Cooperativismo \& Desarrollo ISSN: 0120-7180 ed: Universidad Cooperativa de Colombia

Melián, A. y Campos, V. (2010) Emprendedurismo y economía social como mecanismos de inserción socio-laboral en tiempos de crisis. REVESCO. Revista de Estudios Cooperativos, $\mathrm{N}^{\mathrm{o}} 100$, pp. 43-67.

Monzon, J.L. y Chaves, R. (2012) EESC/CIRIEC. La Economía Social en la Unión Europea. Bruselas: Comité Económico y Social Europeo.

OBSERVATORIO COOPERATIVO. (2012) Comportamiento del sector cooperativo colombiano primer semestre 2012. Bogotá: Confecoop.

OIT Y ACI (2015) Las cooperativas y los Objetivos de Desarrollo Sostenible: Debate sobre el desarrollo después de 2015. Organización Internacional del Trabajo \& Alianza Cooperativa Internacional.

ORGANIZACIONES SOLIDARIAS (Mayo de 2016) www.orgsolidarias.gov.co.

ORGANIZACIONES SOLIDARIAS (s.f) www.orgsolidarias.gov.co. Obtenido de http://www.orgsolidarias.gov.co/sites/default/files/paginabasica/pdf/FondosEmpleados_0.pdf.

Pastor, E. (2011) El papel de la economía social como motor del cambio social y la democratización sostenible de las políticas públicas sociales en el ámbito local. REVESCO. Revista de Estudios Cooperativos, Primer Cuatrimestre, No 104, pp. $143-$ 169. DOI: 10.5209/rev_REVE.2011.v104.5. 
PORTAFOLIO (16 de Octubre de 2014) www.portafolio.co. Obtenido de http://www.portafolio.co/negocios/cooperativas-colombia-impulso-economico-social.

Porter, M. (1991) Ventaja Competitiva de las Naciones. Harvard.

PROCOLOMBIA (2015) Exportaciones totales colombianas según sector - subsector. Bogotá: Procolombia.

Puentes, R. y Velasco, M. M. (2009) Importancia de las sociedades cooperativas como medio para contribuir al desarrollo económico, social y medioambiental, de forma sostenible y responsable. REVESCO. Revista de Estudios Cooperativos, Tercer Cuatrimestre, $\mathrm{N}^{\circ}$ 99, pp, 104-129.

Rodriguez Gaitan, C. C., Rueda Angarita, C. A. y Sánchez Brand, J. E. (2010) Estudio prospectivo de las empresas que se desempeñan dentro del marco de la economía solidaria en Colombia, escenarios al 2010. Chia, Cundinamarca: Universidad de la Sabana.

Sánchez Castañeda, J.M. y Vargas Prieto, A. (2017) Incidencia de las juntas de acción comunal en el desarrollo local en áreas de influencia de proyectos de hidrocarburos. Equidad y Desarrollo, [S.1.], No 27, pp. 195-212, dec. 2016. ISSN 2389-8844. Disponible en: https://revistas.lasalle.edu.co/index.php/ed/article/view/3997. Fecha de acceso: 05 mar. 2017. DOI: http://dx.doi.org/10.19052/ed.3997.

SISTEMA OSB (2015) $\underline{\text { www.brasilcooperativo.coop.br. Obtenido de }}$ http://www.goiascooperativo.coop.br/arquivos/downloads/relatorio-de-atividades-ocbilovepdf-compressed-2141917.pdf.

SUPERSOLIDARIA (ENERO de 2012) Marco legal, funciones y esquemas de supervisión. Obtenido de http://www.supersolidaria.gov.co/es/normativa.

SUPERSOLIDARIA (s.f.) $\underline{\text { www.supersolidaria.gov.co. }}$. Obtenido de http://www.supersolidaria.gov.co/es/nuestra-entidad/resena-historica.

UNIDAD ADMINISTRATIVA DE ORGANIZACIONES SOLIDARIAS (2014) ABC del Sector Solidario. Unidad Administrativa de Organizaciones Solidarias.

Vol. 25, fasc.111, pp. 1-15, 2017, DOI: https://revistas.ucc.edu.co/index.php/co/article/view/1871.

Vargas Prieto, A. (2017) Technical Assistance, Solution for the Identity Crisis of the Agricultural Cooperatives. En: Estados Unidos, Global Advanced Research Journal Of Agricultural Science ISSN: 2315-5094 ed: Vol. 6, fasc.8, pp. 202-214,2017, DOI: http://garj.org/garjas/8/2017/6/8/technical-assistance-solution-for-the-identity-crisis-ofthe-agricultural-cooperatives.

Vargas Prieto, A. (2018) La asociatividad en la construcción de paz en Colombia, en La Colombia del posacuerdo: retos de un país excluido por el conflicto armado/Ricardo García Duarte y otros. Bogotá, Universidad Distrital Francisco José de Caldas, 598 páginas. ISBN 978-958-787-023-7, pp. 403-416. 